\title{
Eficácia da histeroscopia diagnóstica em mulheres na pós menopausa
}

\author{
Efficacy of diagnostic hysteroscopy in postmenopausal women \\ Eficacia de la histeroscopia diagnóstica en mujeres en post menopausia
}

Recebido: 06/01/2022 | Revisado: 11/01/2022 |Aceito: 13/01/2022 | Publicado: 14/01/2022

\author{
Monalisa Cavalcante de Carvalho \\ ORCID: https://orcid.org/0000-0002-5657-0913 \\ Universidade Federal de São Paulo, Brasil \\ E-mail: dramonalisacarvalho@gmail.com \\ Marta Alves Rosal \\ ORCID: https://orcid.org/0000-0003-0680-7772 \\ Universidade Federal do Piauí, Brasil \\ E-mail: mtrosal@hotmail.com
}

\begin{abstract}
Resumo
A histeroscopia diagnóstica é o método padrão-ouro na avaliação endometrial, pois permite visualização direta de toda cavidade uterina, possibilitando a identificação de patologias e biópsia de lesões suspeitas. Avaliar a eficácia da histeroscopia diagnóstica em mulheres na pós-menopausa. Estudo transversal analítico, desenvolvido no Hospital Universitário da UFPI. A amostra foi constituída por 188 pacientes que realizaram histeroscopia diagnóstica no período de setembro de 2014 a setembro de 2017. A histeroscopia foi comparada com a ultrassonografia e anatomopatológico. Foram calculadas estatísticas uni e bivariadas. A idade média das pacientes foi 59,6 $\pm 7,7$ anos, com tempo de menopausa médio de 11,2 \pm 8 anos. A histeroscopia apresentou maior sensibilidade para detecção de pólipo endometrial $(91,8 \%)$ e maior especificidade na exclusão de hiperplasia $(61,4 \%)$. A principal indicação do exame foi espessamento endometrial $(72,3 \%)$ e o achado mais encontrado foi formação polipoide $(45,7 \%)$. Para as pacientes assintomáticas, a ultrassonografia apresentou sensibilidade de $100 \%$ e $100 \%$ e especificidade de $10,6 \%$ e $36,2 \%$ utilizando 5 e $9 \mathrm{~mm}$ como cutoff, respectivamente, para o diagnóstico de hiperplasia atípica/câncer de endométrio. Pacientes com hiperplasia atípica ou câncer apresentaram maior espessura do eco endometrial que as demais $(\mathrm{p}=0,012)$. Não houve correlação entre tempo de sangramento e câncer de endométrio. A histeroscopia foi eficaz no diagnóstico de pólipos endometriais. A principal indicação do exame foi espessamento endometrial, sendo o principal achado histeroscópico o pólipo endometrial. Um maior limite de espessura do eco endometrial pode ser proposto em pacientes menopausadas assintomáticas para a indicação da histeroscopia diagnóstica.
\end{abstract}

Palavras-chave: Ultrassonografia; Histeroscopia; Menopausa.

\begin{abstract}
Diagnostic hysteroscopy is the gold standard method in endometrial evaluation, allowing direct visualization of the entire uterine cavity, besides the identification of pathologies and biopsy of suspicious lesions. To evaluate the efficacy of diagnostic hysteroscopy in postmenopausal women. An analytical cross-sectional study, developed in a university hospital. The sample consisted of 188 patients who underwent diagnostic hysteroscopy from September 2014 to September 2017. Hysteroscopy was compared with ultrasonography and anatomopathological results. Univariate and bivariate statistics were calculated. The mean age of the patients was 59.6 \pm 7.7 years and the mean time of menopause was $11.2 \pm 8$ years. Hysteroscopy showed a higher sensitivity for detection of endometrial polyp (91.8\%) and greater specificity in the exclusion of hyperplasia (61.4\%). The main indication of the examination was endometrial thickening (72.3\%) and the main finding was polypoid formation (45.7\%). For asymptomatic patients, ultrasonography showed $100 \%$ and $100 \%$ of sensitivity and $10.6 \%$ and $36.2 \%$ of specificity using 5 to $9 \mathrm{~mm}$ as cutoff, respectively, for the diagnosis of atypical hyperplasia/endometrial cancer. Patients with atypical hyperplasia or cancer had a greater thickness $(\mathrm{p}=0.012)$. There was no correlation between bleeding time and endometrial cancer. Hysteroscopy was effective in the diagnosis of endometrial polyps. The main indication of the examination was endometrial thickening and the main hysteroscopic finding was endometrial polyp. Greater limit of endometrial echo thickness can be proposed in asymptomatic postmenopausal women for the indication of hysteroscopy.
\end{abstract}

Keywords: Ultrasonography; Hysteroscopy; Menopause.

\section{Resumen}

La histeroscopia diagnóstica es el método de referencia en la valoración del endometrio, ya que permite la visualización directa de toda la cavidad uterina, lo que permite la identificación de patologías y la biopsia de lesiones sospechosas. Evaluar la efectividad de la histeroscopia diagnóstica en mujeres posmenopáusicas. Estudio analítico transversal, desarrollado en el Hospital Universitario de la UFPI. La muestra estuvo constituida por 188 pacientes que fueron sometidas a histeroscopia diagnóstica desde septiembre de 2014 a septiembre de 2017. Se comparó la 
histeroscopia con la ecografía y el examen patológico. Se calcularon estadísticas uni y bivariadas. La edad media de las pacientes fue de 59,6 \pm 7,7 años, con un tiempo medio desde la menopausia de 11,2 \pm 8 años. La histeroscopia mostró mayor sensibilidad para detectar pólipos endometriales $(91,8 \%)$ y mayor especificidad para excluir hiperplasia $(61,4 \%)$. La principal indicación para la exploración fue el engrosamiento endometrial $(72,3 \%)$ y el hallazgo más frecuente fue la formación de polipoides $(45,7 \%)$. Para los pacientes asintomáticos, la ecografía tuvo una sensibilidad del $100 \%$ y el $100 \%$ y una especificidad del $10,6 \%$ y el $36,2 \%$ utilizando 5 y $9 \mathrm{~mm}$ como punto de corte, respectivamente, para el diagnóstico de hiperplasia atípica / cáncer de endometrio. Los pacientes con hiperplasia atípica o cáncer tenían mayor grosor del eco endometrial que los demás $(\mathrm{p}=0,012)$. No hubo correlación entre el tiempo de sangrado y el cáncer de endometrio. La histeroscopia fue eficaz en el diagnóstico de pólipos endometriales. La principal indicación para la exploración fue el engrosamiento endometrial, siendo el principal hallazgo histeroscópico el pólipo endometrial. Se puede proponer un límite de espesor del eco endometrial más alto en pacientes posmenopáusicas asintomáticas para la indicación de histeroscopia diagnóstica.

Palabras clave: Ecografía; Histeroscopia; Menopausia.

\section{Introdução}

A menopausa, termo que corresponde à última menstruação feminina, traduz a falência ovariana e corresponde a um evento dentro do período do climatério. Este último, por sua vez, marca a transição entre o período reprodutivo e o não reprodutivo feminino. A idade de ocorrência da menopausa é variável, mas ocorre em média aos 50 anos (Baracat et al.,2003).

De acordo com os resultados da revisão da Organização Mundial de Saúde (WHO) (World Health Organization, 2017), a população mundial está próxima de 7,6 bilhões de habitantes, sendo $13 \%$ destes acima de 60 anos. A expectativa para 2050 é de uma população mundial de 9,78 bilhões, com 2,1 bilhões de pessoas acima dos 60 anos e na sua maioria do sexo feminino (World Health Organization, 2017).

Com a maior longevidade, as mulheres podem viver um terço de suas vidas na pós-menopausa. Consequentemente estão sujeitas às afecções próprias dessa fase, como as neoplasias malignas dos órgãos genitais, e dentre elas, o câncer de endométrio, que representa a principal neoplasia do trato genital feminino nos países desenvolvidos (Baracat et al.,2003). No ano de 2017 foram estimados 56470 novos casos de câncer de endométrio nos Estados Unidos (EUA) (American Cancer Society, 2017). No Brasil, esta patologia ocupa o sexto lugar entre as neoplasias malignas da população feminina, excetuandose os tumores de pele não melanoma. O registro de base populacional da incidência ajustada é 6,74 casos para cada 100.000 mulheres (INCA, 2015).

A maioria dos casos de câncer de endométrio apresenta-se em mulheres acima dos 50 anos. Apenas $15 \%$ dos casos diagnosticados estão presentes em mulheres abaixo dessa faixa etária e, desses, somente 5\% abaixo dos 40 anos (Instituto Nacional de Câncer José Alencar Gomes da Silva, 2015). A idade precoce da menarca, menopausa tardia, terapia de reposição hormonal (TRH) e obesidade são os principais fatores de risco. Outros fatores estudados são diabetes mellitus e hipertensão arterial (American Cancer Society, 2017; INCA, 2015).

O sangramento transvaginal é a principal manifestação clínica do carcinoma endometrial. Entretanto, uma parte das pacientes com essa patologia é assintomática (American Cancer Society, 2017; INCA, 2015; Worley et al.,2011). O risco de câncer de endométrio nas pacientes menopausadas sem sangramento transvaginal (assintomáticas) é de 10\% (Gambacciani et al.,2004).

O rastreamento dessa neoplasia maligna tem sido amplamente discutido e existem vários trabalhos divergentes na literatura, tanto em relação às mulheres que devem ser avaliadas quanto ao método diagnóstico a ser utilizado (Donadio \& Albuquerque, 2001).

Por muito tempo a dilatação e curetagem uterina foram utilizadas como métodos de escolha para diagnóstico de câncer de endométrio, porém eles possuem taxas de falso negativos que variam de 2 a 10\% (Tinelli et al.,2008).

A ultrassonografia, apesar de não ser método de rastreio para câncer de endométrio, é amplamente utilizada na investigação de pacientes com sangramento uterino na pós menopausa. É um método não invasivo, de fácil acesso, baixo custo 
e maior conforto para a paciente (World Health Organization, 2017).

A medida da espessura do eco endometrial (EE) é um dos parâmetros avaliados por meio da ultrassonografia transvaginal (USTV) na investigação de patologias da cavidade uterina. A espessura do eco endometrial de 4-5 mm nas pacientes sem o uso de terapia de reposição hormonal (TRH) e 8-10 mm nas pacientes em uso de TRH são adotadas como limites de normalidade. Acima destes valores, a cavidade uterina deve ser investigada em pacientes com sangramento transvaginal (sintomáticas) na pós menopausa (Gambacciani et al.,2004). Porém, não existe consenso quanto ao limite a ser utilizado em paciente assintomáticas (ACOG, 2009).

Ainda que a USTV apresente substancial importância na investigação do carcinoma endometrial, nem sempre espessura de eco endometrial acima do normal corresponde a alterações reais da mucosa (Campaner et al.,2004). Isso ocorre porque a USTV não permite a diferenciação inequívoca das imagens endometriais entre pólipos, hiperplasias e fenômenos proliferativos resultantes de terapia hormonal (Machado et al.,2003).

Com o desenvolvimento das tecnologias em saúde, alguns procedimentos endoscópicos foram desenvolvidos, dispondo-se hoje da histeroscopia diagnóstica, que promove visão direta da cavidade uterina. Permite-se, ainda, a realização de biópsias dirigidas e, por conseguinte, melhora a acurácia no diagnóstico de lesões intra-uterinas, especialmente as focais (Donadio \& Albuquerque, 2001). A histeroscopia diagnóstica é considerada o método padrão-ouro no diagnóstico de doenças endometriais. Porém, por se tratar de método invasivo, os critérios para sua indicação tem sido motivo de discussão na literatura (Costa \& Costa, 2008).

Em virtude da grande relevância do tema, esta pesquisa foi realizada tendo como objetivo geral avaliar a eficácia da histeroscopia diagnóstica em mulheres na pós menopausa e como objetivos específicos avaliar as indicações desse exame associando-as com a frequência dos achados histeroscópicos, correlacionar os achados histeroscópicos com os resultados do anatomopatológico e estimar a sensibilidade e especificidade da espessura do eco endometrial no diagnóstico de hiperplasia atípica/câncer de endométrio em pacientes assintomáticas.

\section{Metodologia}

O delineamento transversal analítico, com avaliação preditiva de métodos diagnósticos, foi realizado no Hospital Universitário da Universidade Federal do Piauí (UFPI), conforme a metodologia de Estrela (2018). A população do estudo correspondeu a 329 mulheres que estavam na pós- menopausa e que foram submetidas à histeroscopia diagnóstica no setor de ginecologia do hospital, no período de setembro de 2014 a setembro de 2017. A menopausa foi definida como ausência de menstruação por um período mínimo de 12 meses (Berek, 2014).

Foram excluídas 141 pacientes pelos seguintes motivos: histeroscopia interrompida antes da conclusão definitiva (20), presença de sangramento uterino no momento do exame (1), câncer ginecológico previamente diagnosticado (2), resultado histopatológico inconclusivo (2) e achados histeroscópicos de pólipo endometrial, espessamento, sugestivo de hiperplasia ou sugestivo de neoplasia, mas com ausência de histopatológico (116). A amostragem foi não probabilística, do tipo por conveniência, e ficou composta por 188 mulheres.

Para coleta de dados, foi utilizado um formulário contendo características sociodemográficas e de saúde (idade, tempo de menopausa, número de gestações, partos e abortos, tabagismo atual, tabagismo prévio, hipertensão arterial sistêmica, diabetes mellitus, terapia de reposição hormonal, uso de tamoxifeno e neoplasias). Foram registrados a indicação da histeroscopia, resultado da ultrassonografia pélvica, da histeroscopia e do histopatológico. Para a caracterização dos diferentes achados histeroscópicos utilizou-se como base a classificação de Labastida (Labastida, 1990).

Os exames histeroscópicos foram realizados em caráter ambulatorial no setor de ginecologia do hospital universitário e seguiram os seguintes padrões: ópticas 4,0e 2,9 mm com angulação de 30 graus, da marca STORZ®; camisas diagnósticas 
circular e de Betochi; os meios de distensão foram soro fisiológico $0,9 \%$ ou gás carbônico $\left(\mathrm{CO}_{2}\right)$. A pressão da cavidade uterina foi mantida entre 70 a $100 \mathrm{mmHg}$ através de um insuflador. Padronizou-se o inventário da cavidade uterina nos seguintes tempos: parede uterina anterior, óstio tubário direito e parede lateral direita, óstio tubário esquerdo e parede lateral esquerda, parede uterina posterior, panorâmica da cavidade, avaliação do canal cervical. As biópsias foram obtidas por meio de cureta de Novak, acoplada a seringa descartável de $20 \mathrm{~mL}$. Os materiais obtidos foram colocados em frascos contendo solução de formaldeído 10\%. Todas as biópsias foram encaminhadas ao setor de patologia e o laudo foi emitido de acordo com protocolos institucionais. Foi utilizado o prontuário eletrônico para identificação das mulheres que atendiam aos critérios de inclusão e para obtenção dos dados para preenchimento do formulário da pesquisa. Pacientes cujos achados na histeroscopia foram mioma, sinéquia, muco, distrofia vascular, áreas enegrecidas ou septo dispensaram o resultado do anatomopatológico.

Os dados foram processados no software IBM® SPSS®, versão 21.0, para cálculo de estatísticas descritivas, como médias, medianas, desvio padrão, mínimos e máximos, para as variáveis quantitativas; e frequências, para as qualitativas. Na análise inferencial, para as variáveis independentes qualitativas foi utilizado o Teste Exato de Fisher para verificação de associações; para as independentes quantitativas, a normalidade dos dados foi verificada por meio do Teste de KolmogorovSmirnov e foi realizado o Teste de Mann-Whitney.

$\mathrm{Na}$ análise de acurácia, foi utilizado o software Analyse-it®, integrado ao Microsoft Excel®, para cálculo de medidas de qualidade (sensibilidade, especificidade, valores preditivos positivo e negativo, razões de verossimilhança positiva e negativa) e caracterização das propriedades preditivas (precisão, eficiência e coeficiente phi de correlação de Matthews), tomando como padrão-ouro o resultado do anatomopatológico.

Os dados de predição dos métodos diagnósticos foram utilizados para construção da curva Receiver Operating Characteristic (ROC) e verificação da Area Under the Curve (AUC). Para o cálculo das propriedades preditivas, foram construídas tabelas de contingência, utilizando-se as variáveis dicotomizadas dos achados "pólipo endometrial", "hiperplasia/espessamento" e "neoplasia de endométrio" nos laudos de histeroscopia e anatomopatológico. Todas as análises foram realizadas ao nível de significância de 5\%.

Foram atendidas as normatizações éticas nacionais e internacionais para realização de pesquisas envolvendo seres humanos. A pesquisa foi autorizada pelo hospital universitário e foi aprovada por Comitê de Ética, sob parecer n. $^{\circ}$ 2.366.482/2017.

\section{Resultados}

A média de idade das mulheres foi de 59,6 $( \pm 7,7)$ anos, com tempo de menopausa médio de 11,2 $( \pm 8,0)$ anos, sendo $3,2 \%(\mathrm{n}=6)$ tabagistas, $42,6 \%(\mathrm{n}=80)$ hipertensas, $12,2 \%(\mathrm{n}=23)$ diabéticas. Terapia de reposição hormonal foi realizada por 12 $(6,4 \%)$ pacientes e tamoxifeno por $3(1,6 \%)$. Foram $5(2,7 \%)$ as que apresentaram neoplasias, destacando-se as de mama 4 $(2,1 \%)$ e Linfoma de Hodgkin 1 (0,5\%).

A indicação de histeroscopia diagnóstica mais prevalente foi o espessamento endometrial, presente em 136 casos (72,3\%), seguido do sangramento transvaginal na pós-menopausa em 71 casos (37,8\%). Os principais achados histeroscópicos foram formação polipoide endometrial em $45,7 \%$ dos casos $(n=86)$, seguido de espessamento endometrial em 18,1\% ( $\mathrm{n}=34)$.

Pacientes cuja indicação da histeroscopia foi espessamento endometrial, seja focal ou difuso, houve relação estatisticamente significativa com os achados de sinéquias e pólipo endometrial ( $\mathrm{p}=0,044$ e $\mathrm{p}=0,001$, respectivamente). Foi verificado significância estatística entre os achados de pólipo endometrial e imagem sugestiva de neoplasia em mulheres cuja indicação da histeroscopia foi presença de líquido intracavitário à ultrassonografia. Tais pacientes também apresentavam espessamento endometrial ao ultrassom (Tabela 1). 
Tabela 1. Distribuição de frequências das indicações e achados das histeroscopias diagnósticas realizadas em mulheres na pósmenopausa ( $\mathrm{n}=188)$. Teresina, PI, Brasil, 2017.

\begin{tabular}{|c|c|c|c|c|c|c|c|c|c|c|c|c|}
\hline \multirow[t]{3}{*}{ Indicações } & \multicolumn{12}{|c|}{ Achados } \\
\hline & \multicolumn{2}{|c|}{ Mioma } & \multicolumn{2}{|c|}{ Sinéquia } & \multicolumn{2}{|c|}{ Pólipo } & \multicolumn{2}{|c|}{$\mathbf{E}$} & \multicolumn{2}{|c|}{$\mathbf{H}$} & \multicolumn{2}{|r|}{$\mathbf{C}$} \\
\hline & $\mathbf{n}$ & $\%$ & $\mathbf{n}$ & $\%$ & $\mathbf{n}$ & $\%$ & $\mathbf{n}$ & $\%$ & $\mathbf{n}$ & $\%$ & $\mathbf{n}$ & $\%$ \\
\hline Sangramento, sem E & 3 & 1,6 & 5 & 2,7 & 6 & 3,2 & 2 & 1,1 & 1 & 0,5 & - & - \\
\hline p valor & \multicolumn{2}{|c|}{0,431} & \multicolumn{2}{|c|}{0,198} & \multicolumn{2}{|c|}{0,325} & \multicolumn{2}{|c|}{0,536} & \multicolumn{2}{|c|}{1,000} & \multicolumn{2}{|c|}{1,000} \\
\hline $\begin{array}{l}\text { Espessamento focal ou } \\
\text { Difuso }\end{array}$ & 17 & 9,0 & 24 & 12,8 & 82 & 43,6 & 30 & 16,0 & 13 & 6,9 & 1 & 0,5 \\
\hline$p$ valor & \multicolumn{2}{|c|}{0,491} & \multicolumn{2}{|c|}{0,044} & \multicolumn{2}{|c|}{0,001} & \multicolumn{2}{|c|}{1,000} & \multicolumn{2}{|c|}{0,222} & \multicolumn{2}{|c|}{1,000} \\
\hline Líquido intracavitário & - & - & 2 & 1,1 & 1 & 0,5 & 3 & 1,6 & - & - & 1 & 0,5 \\
\hline p valor & \multicolumn{2}{|c|}{0,601} & \multicolumn{2}{|c|}{0,652} & \multicolumn{2}{|c|}{0,041} & \multicolumn{2}{|c|}{0,209} & \multicolumn{2}{|c|}{1,000} & \multicolumn{2}{|c|}{0,048} \\
\hline Hematométrio & - & - & - & - & - & - & - & - & - & - & - & - \\
\hline p valor & \multicolumn{2}{|c|}{1,000} & \multicolumn{2}{|c|}{1,000} & \multicolumn{2}{|c|}{1,000} & \multicolumn{2}{|c|}{1,000} & \multicolumn{2}{|c|}{1,000} & & 000 \\
\hline Calcificação & - & - & - & - & - & - & - & - & 1 & 0,5 & - & - \\
\hline p valor & \multicolumn{2}{|c|}{1,000} & \multicolumn{2}{|c|}{1,000} & \multicolumn{2}{|c|}{0,501} & \multicolumn{2}{|c|}{1,000} & \multicolumn{2}{|c|}{0,134} & & 000 \\
\hline Total & 21 & 11,2 & 32 & 17,0 & 86 & 45,7 & 34 & 18,1 & 13 & 6,9 & 2 & 1,1 \\
\hline
\end{tabular}

Legenda: E: espessamento; H: hiperplasia; C: imagem sugestiva de câncer; $p$ : significância do TesteExato de Fisher Fonte: Hospital Universitário (HU-UFPI) (2017).

Quanto aos achados histopatológicos, verificou-se em 44,95\% (n=49) a presença de pólipo, 21,1\% ( $\mathrm{n}=23$ ) endométrio sem atipias, $0,09 \%(n=1)$ endométrio atrófico, em 1,83\% $(n=2)$ hiperplasia e outros 6,42\% ( $n=7)$ carcinoma. Todos os carcinomas histopatologicamente diagnosticados não foram corretamente identificados por histeroscopia, sendo em 2 casos atribuídos o diagnóstico de pólipo, 2 casos de espessamento endometrial e em 3 de hiperplasia.

Dentre as mulheres com espessamento endometrial à ultrassonografia, comparando a espessura do endométrio naquelas que tiveram queixa de sangramento transvaginal ou não, verificou-se médias de $12,5 \mathrm{~mm}( \pm 7,9)$ e de $9,8 \mathrm{~mm}( \pm 5,4)$, respectivamente, e medianas de $10,8 \mathrm{~mm}$ e $9,0 \mathrm{~mm}$, nesta ordem, com diferença estatisticamente significativa entre as médias $(\mathrm{p}=0,041)$.

Estratificando as mulheres assintomáticas com espessamento endometrial no cutoff 9,0 mm, com base na mediana, todos os casos de câncer de endométrio 2 (3,3\%) foram acima dessa medida. A ultrassonografia apresentou sensibilidade de $100 \%$ e $100 \%$ e especificidade de $10,6 \%$ e $36,2 \%$ utilizando 5 e 9 mm como cutoff, respectivamente (Tabela 2).

Tabela 2. Sensibilidade e especificidade das medidas do eco endometrial espessado em mulheres na pós-menopausa sem sangramento vaginal com diagnóstico de carcinoma/ hiperplasia atípica no anatomopatológico.

\begin{tabular}{lccccc}
\hline Propriedade* & \multicolumn{5}{c}{ Cutoff $(\mathbf{I C = 9 5 \% )}$} \\
\cline { 2 - 6 } & $\mathbf{5}$ & $\mathbf{6}$ & $\mathbf{7}$ & $\mathbf{8}$ & $\mathbf{9}$ \\
\hline Sensiblidade & 100,0 & 100,0 & 100,0 & 100,0 & 100,0 \\
Especificidade & 10,6 & 19,1 & 25,5 & 36,2 & 36,2 \\
\hline
\end{tabular}

Legenda: IC: Intervalo de confiança; e: cutoff estatístico; *: expressos em \%.

Fonte: Hospital Universitário (HU-UFPI) (2017).

A sensibilidade (S) das histeroscopias foi maior para detecção de pólipo endometrial $(91,8 \%)$ e a especificidade (E) foi superior na exclusão de mulheres que não possuem hiperplasia (61,4\%). Embora para o comparativo dos resultados de neoplasias a especificidade tenha sido $99,0 \%$, não foram gerados valores para sensibilidade, valor preditivo positivo (VPP) e 
razão de verossimilhança positiva (RVP), com ausência de concordância entre resultados de presença de neoplasias. O coeficiente de correlação de Mathews indicou baixa qualidade de predição das histeroscopias diagnósticas nas mulheres na pós-menopausa (Tabela 3).

Tabela 3. Propriedades preditivas das histeroscopias diagnósticas comparadas aos exames anatomopatológicos das mulheres na pós-menopausa (n=188). Teresina, PI, Brasil, 2017.

\begin{tabular}{|c|c|c|c|}
\hline \multirow[t]{2}{*}{ Propriedade } & \multicolumn{3}{|c|}{ Valor } \\
\hline & $\begin{array}{c}\text { Pólipo }^{1} \mathrm{x} \\
\text { Pólipo }^{2}\end{array}$ & H/E $\mathbf{E}^{1} \times$ Hiperplasia ${ }^{2}$ & $\begin{array}{c}\text { Sugestivo de neoplasia } \\
\text { x Carcinoma }^{2}\end{array}$ \\
\hline Sensiblidade* & 91,8 & 38,5 & 0,0 \\
\hline Especificidade* & 38,3 & 61,4 & 99,0 \\
\hline Valor preditivo positivo* & 54,9 & 11,9 & 0 \\
\hline Valor preditivo negativo* & 85,2 & 88,1 & 93,5 \\
\hline $\begin{array}{l}\text { Razão de verossimilhança } \\
\text { positiva }\end{array}$ & 1,49 & 0,99 & 0,00 \\
\hline $\begin{array}{l}\text { Razão de verossimilhança } \\
\text { negativa }\end{array}$ & 0,21 & 1,00 & 1,00 \\
\hline Precisão & 62,4 & 58,7 & 92,6 \\
\hline Eficiência & 65,1 & 49,9 & 49,5 \\
\hline Coeficiente $\varphi$ de Mathews & 0,348 & $-0,00053$ & $-0,025$ \\
\hline
\end{tabular}

Legenda: *: expresso em \%; H/E: hiperplasia ou espessamento; ${ }^{1}$ : achado histeroscópico; ${ }^{2}$ :resultado do histopatológico Fonte: Hospital Universitário (HU-UFPI) (2017).

Os valores de sensibilidade e especificidade das histeroscopias diagnósticas para os três achados avaliados (pólipo, hiperplasia/espessamento e neoplasia) formaram uma área abaixo da curva Receiver Operating Characteristic de 0,651 (IC95\%=0,578-0,724), 0,500 (IC95\%=0,354-0,646) e 0,505 (IC95\%=0,495-0,515), respectivamente, indicando discriminação fraca para os três achados, porém com melhor valor para o diagnóstico de pólipo endometrial (Gráfico 1). 
Gráfico 1. Curvas Receiver Operating Characteristic e Areas Under the Curve da predição das histeroscopias comparativamente ao anatomopatológico das mulheres na pós-menopausa (n=188). Teresina, PI, Brasil, 2017.

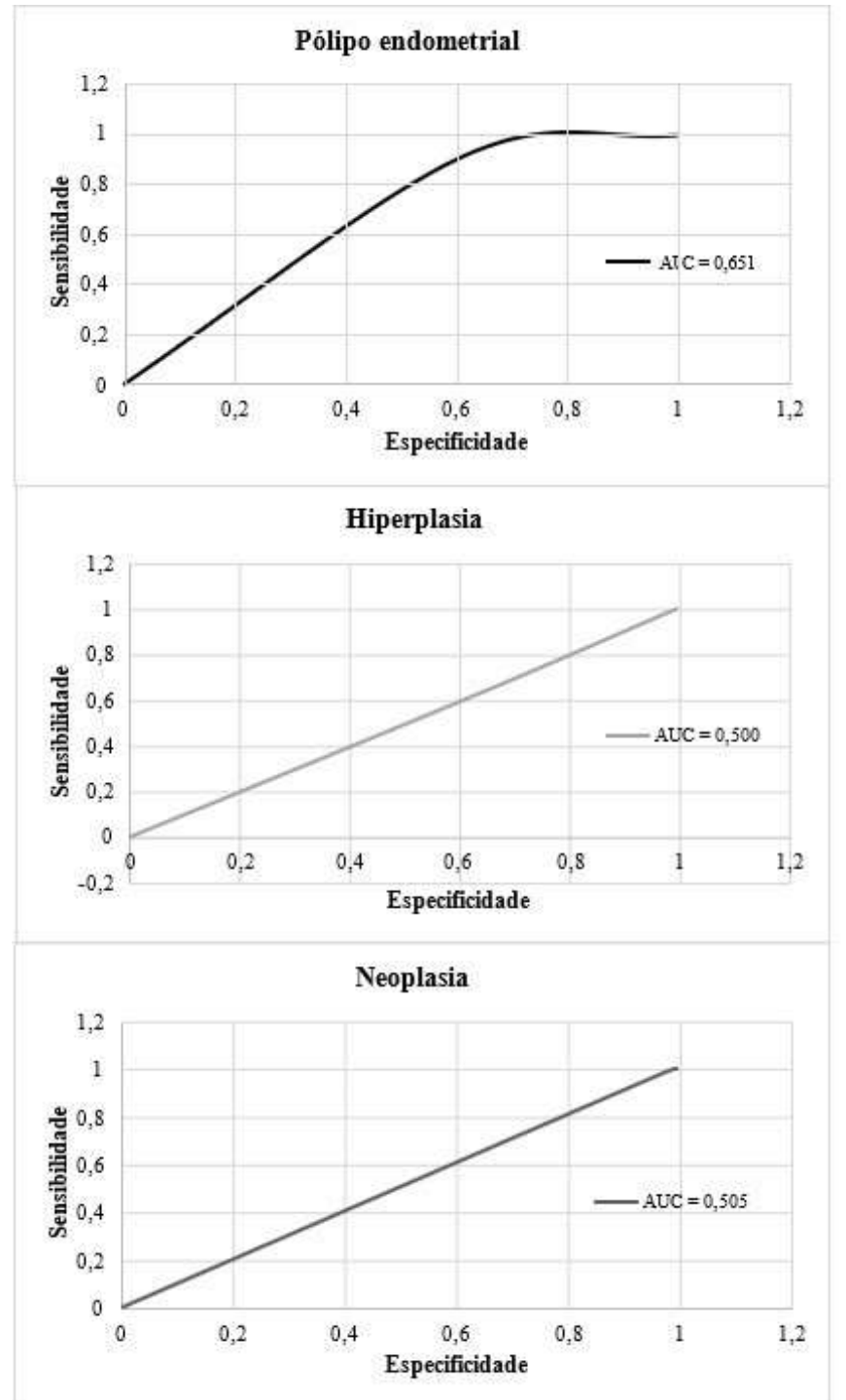

Legenda: AUC: Area Under the Curve. Fonte: Hospital Universitário (HU-UFPI), 2017.

Foi verificada diferença estatisticamente significativa entre as distribuições dos graus de espessamento endometrial entre as mulheres que apresentavam lesões endometriais pré-malignas ou malignas comparadas as demais pacientes, porém não foram encontradas diferenças entre as distribuições dos demais parâmetros analisados (Tabela 4). Estratificando apenas o grupo de pacientes com sangramento vaginal, não foi observado correlação entre o tempo de sangramento e o desenvolvimento de câncer de endométrio, porém, quantitativamente, as pacientes com neoplasia endometrial apresentavam maior tempo de sangramento (em meses) que as demais $(11,25 \pm 9,28$ versus $7,32 \pm 8,59, \mathrm{p}=0,224)$. 
Tabela 4. Distribuição das mulheres na pós-menopausa conforme presença de hiperplasia atípica ou carcinoma de endométrio no anatomopatológico $(\mathrm{n}=109)$. Teresina, PI, Brasil, 2017

\begin{tabular}{|c|c|c|c|c|c|c|c|c|c|}
\hline \multirow{3}{*}{ Característica } & \multicolumn{8}{|c|}{ Hiperplasia atípica ou câncer de endométrio } & \multirow{3}{*}{$\boldsymbol{P}$} \\
\hline & \multicolumn{3}{|c|}{ Sim } & & \multicolumn{4}{|c|}{ Não } & \\
\hline & $\mathbf{M}$ & DP & $\mathbf{n}$ & $\%$ & $\mathbf{M}$ & DP & $\mathbf{n}$ & $\%$ & \\
\hline Idade & 59,6 & 5,4 & & & 60,0 & 6,8 & & & $0,952^{\mathrm{u}}$ \\
\hline Tempo de menopausa & 10,7 & 8,7 & & & 11,3 & 8,2 & & & $0,843^{\mathrm{u}}$ \\
\hline Gestações & 5,9 & 3,3 & & & 4,4 & 3,3 & & & $0,138^{\mathrm{u}}$ \\
\hline Partos & 5,4 & 3,3 & & & 4,1 & 2,9 & & & $0,183^{\mathrm{u}}$ \\
\hline Abortos & 0,4 & 0,5 & & & 0,4 & 0,8 & & & $0,255^{\mathrm{u}}$ \\
\hline Tabagismo atual & & & 1 & 0,9 & & & 3 & 2.8 & $0,295^{f}$ \\
\hline Tabagismo prévio & & & 0 & 0,0 & & & 16 & 14,7 & $0,351^{\mathrm{f}}$ \\
\hline $\begin{array}{l}\text { Hipertensão } \\
\text { arterial }\end{array}$ & & & 4 & 3,7 & & & 53 & 48,6 & $0,734^{f}$ \\
\hline Diabetes mellitus & & & 1 & 0,9 & & & 15 & 13,8 & $1,000^{f}$ \\
\hline $\begin{array}{l}\text { Terapia de reposição } \\
\text { hormonal }\end{array}$ & & & 1 & 0,9 & & & 7 & 6,4 & $0,510^{f}$ \\
\hline Tamoxifeno & & & 0 & 0,0 & & & 1 & 0,9 & $1,000^{f}$ \\
\hline Neoplasias & & & 0 & 0,0 & & & 2 & 1,8 & $1,000^{f}$ \\
\hline $\begin{array}{l}\text { Valor do } \\
\text { espessamento } \\
\text { endometrial } \\
\end{array}$ & 17,9 & 8,9 & & & 10,9 & 6,2 & & & $0,012^{\mathrm{u}}$ \\
\hline Total & & & 9 & 8,3 & & & 100 & 91,7 & \\
\hline
\end{tabular}

Legenda: $u$ : Teste de Mann-Whitney; $f$ : Teste Exato de Fisher; M: média; DP: desvio padrão Fonte: Hospital Universitário (HU-UFPI), 2017

\section{Discussão}

A histeroscopia diagnóstica tem sido largamente utilizada na investigação de anormalidades na cavidade uterina, pois possibilita visão direta das lesões endometriais e a realização de biópsias dirigidas (sob visão direta no momento do exame) ou orientadas (imediatamente após o exame, de acordo com a orientação obtida pela imagem) (Machado et al., 2003; Costa \& Costa, 2008; Branco et al., 2008).

No presente estudo, o principal achado das histeroscopias diagnósticas foi pólipo endometrial, o que foi concordante com a literatura. Em estudo transversal, Sarvi et al. (2016) avaliaram 110 mulheres na pós menopausa e encontraram pólipo endometrial em $44,1 \%$ e 53,5\% das pacientes, com e sem sangramento transvaginal, respectivamente. Metello et al. (2008) mostraram frequência de 45,5\% para pólipo endometrial em pesquisa envolvendo 335 mulheres com sangramento transvaginal pós menopausa.

Quando a indicação da histeroscopia foi espessamento endometrial, houve correlação com o achado de pólipo endomtrial no exame. Na literatura também se observam resultados semelhantes (Campaner et al., 2004; Branco et al., 2008; Sarvi et al., 2016, Yela et al., 2018).

Foi verificado associação entre os achados histeroscópicos de pólipo endometrial e imagem sugestiva de câncer com a presença de líquido intracavitário à ultrassonografia. Tal achado, entretanto, constitui um viés do estudo, visto que tais pacientes possuíam também espessamento endometrial. Topçu et al. (2015) analisaram o endométrio de 141 pacientes na pós menopausa com presença de líquido intracavitáro na USTV e não foi encontrada nenhuma patologia pré maligna ou maligna. Takacs et al (2005) analisando retrospectivamente os achados histopatológicos de 343 mulheres menopausadas com líquido 
intracavitário ao exame ultrassonográfico, não observaram nenhum caso de neoplasia de endométrio em pacientes com espessura de eco endometrial inferior à 3mm e concluíram que a investigação da cavidade uterina naquele grupo de pacientes deve ser realizada apenas quando a espessura do eco endometrial for superior a $3 \mathrm{~mm}$ ou o líquido for ecogênico.

Quanto aos resultados histopatológicos, observou-se 6,42\% ( $\mathrm{n}=7$ ) casos de câncer de endométrio, 1,83\% ( $\mathrm{n}=2)$ de hiperplasia endometrial e 44,95\% (n=49) de pólipo endometrial. Na literatura, estas porcentagens são semelhantes às deste estudo. Em estudo transversal, Yela et al., (2018) avaliaram 243 mulheres na pós menopausa e observaram 3\% de câncer de endométrio, 3,6\% de hiperplasia endometrial e 54\% de pólipo endometrial. Em análise transversal de 148 mulheres menopausadas, Pop-Trajkovic-Dinic et al (2013) encontraram 3\%, 8\% e 47\%, respectivamente.

A sensibilidade (S) das histeroscopias foi maior para detecção de pólipo endometrial $(91,8 \%)$. Em estudo retrospectivo com 281 mulheres na pós menopausa, Borges (2010) obteve resultados semelhantes, com sensibilidade 96,4\% e especificidade 74,6\% da histeroscopia no diagnóstico de pólipos endometriais.

$\mathrm{O}$ fato de todas as biópsias realizadas neste estudo terem sido orientadas pela histeroscopia diagnóstica pode explicar o baixo valor preditivo positivo do procedimento para o diagnóstico de pólipos endometriais, já que essas patologias são focais e, portanto, melhor diagnosticadas por biópsias dirigidas. Para que não ocorram discrepâncias entre o achado histeroscópico e o exame histopatológico, é de fundamental importância que a biópsia seja realizada pelo método mais apropriado, isto é, a técnica dirigida (Tinelli et al., 2008; Costa \& Costa, 2008). Na literatura, as taxas de falhas com o emprego de biópsias pela técnica orientada variam em torno de 10-30\% (Machado et al., 2003; Accorsi et al., 2003).

Todos os sete carcinomas histopatologicamente diagnosticados neste estudo não foram corretamente identificados por histeroscopia. Na literatura, há heterogeneidade de resultados. Metello et al. (2008) verificaram sensibilidade e especificidade da histeroscospia para o diagnóstico de câncer de endométrio de 81,3 e 98,7\%. Sarvi et al. (2016) apresentaram sensibilidade 100\% e especificidade $97 \%$. Entretanto, outros estudos similares mostraram baixa sensibilidade e valores preditivos positivos da histeroscopia no diagnóstico desta patologia. Lo e Yuen (2000) apresentaram sensibilidade e valor preditivo positivo de $58,8 \%$ e $20,8 \%$, respectivamente. Elfayomy et al. (2012) obtiveram sensibilidade $50 \%$, especificidade $94,2 \%$, valor preditivo positivo $63,6 \%$ e valor preditivo negativo $90,2 \%$. O mesmo estudo mostrou que $20 \%$ dos pólipos tinham componentes de malignidade no seu centro, o que pode justificar a incapacidade de identificação visual do carcinoma nessa pesquisa, concluindo o papel limitado da histeroscopia sem biópsia no diagnóstico carcinoma de endométrio. Além disso, a discordância entre os resultados da histeroscopia e histologia pode estar relacionada à experiência do examinador em interpretar os achados histeroscópicos e obter biópsias de forma correta.

Foram nove o número de pacientes diagnosticados com hiperplasia atípica ou câncer de endométrio e a média de espessura endometrial deste grupo foi significativamente maior que as demais pacientes do estudo $(17,9 \pm 8,9 \mathrm{~mm}$ versus 10,9 $\pm 6,2 \mathrm{~mm}, \mathrm{p}=0,012$ ). Silva (2015), em estudo transversal envolvendo 1952 mulheres na pós menopausa, mostrou uma média de espessura de endométrio significativamente superior nas pacientes com hiperplasia atípica/câncer de endométrio, comparadas às demais $(16,4 \mathrm{~mm}$ versus $11,8 \mathrm{~mm})$.

Sangramento transvaginal é o sintoma mais comum de câncer de endométrio. Entretanto, existem poucos dados na literatura correlacionando o tempo de sangramento transvaginal com o desenvolvimento desta patologia. Nesta pesquisa, não foi observado correlação entre o tempo de sangramento e o desenvolvimento de câncer de endométrio, porém, quantitativamente, as pacientes com neoplasia endometrial apresentavam maior tempo de sangramento que as demais. Em um estudo prospectivo envolvendo 3047 mulheres na pós menopausa com sangramento transvaginal, Burbos et al. (2010) evidenciaram que pacientes com episódios recorrentes de sangramento transvaginal tinham maior risco de desenvolvimento de câncer de endométrio, porém o volume de sangramento não foi associado com o aumento do mesmo.

O risco de câncer de endométrio em pacientes assintomáticas é baixo (Gambacciani et al., 2004; Breijer et al., 2012). 
Neste estudo, foram diagnosticados dois casos de carcinoma de endométrio em pacientes assintomáticas com espessamento endometrial à USTV. Ainda não foi validado um limite de espessura de eco endometrial acima do qual a cavidade uterina deva ser investigada em pacientes menopausadas sem sangramento transvaginal (ACOG, 2009). Smith-Bindman et al. (2004) propuseram o limite de $11 \mathrm{~mm}$ para indicar histeroscopia diagnóstica em pacientes assintomáticas baseado em um modelo matemático que estima o risco de câncer de endométrio de 6,7\% nestas pacientes, semelhante ao risco das pacientes com sangramento transvaginal. A metanálise realizada por Breijer et al (2012), que reuniu 32 estudos com 11100 mulheres na pós menopausa, indicou inacurácia da espessura do eco endometrial medida pelo USTV no diagnóstico de câncer endometrial, devido à baixa prevalência da doença em pacientes menopausadas assintomáticas. No presente estudo, utilizando maior limite de espessura de eco endometrial $(9 \mathrm{~mm})$, aumentou-se a especificidade da USTV no diagnóstico de câncer em pacientes assintomáticas, quando comparado ao cutoff de $5 \mathrm{~mm}$. Porém, esse valor por si só, não é capaz de justificar a não investigação de casos de espessamento endometrial superiores a $5 \mathrm{~mm}$, pois trata-se de um estudo transversal e o número de pacientes assintomáticas nesta pesquisa foi limitado.

Por fim, é oportuno salientar que a pesquisa teve como limitação principal a perda de considerável quantidade de pacientes devido à falta de resultado de anatomopatológico, fato que pode ter prejudicado os resultados do estudo.

\section{Conclusão}

A histeroscopia mostrou-se eficaz na avaliação endometrial de mulheres na pós menopausa quando o diagnóstico foi pólipo endometrial. A principal indicação do exame foi espessamento endometrial à ultrassonografia e o principal achado histeroscópico foi pólipo endometrial. Um maior limite de espessura do eco endometrial pode ser proposto em pacientes menopausadas assintomáticas para indicação da histeroscopia diagnóstica, porém novos estudos prospectivos e com uma maior casuística devem ser realizados para validar essa proposta.

Estudos desta natureza podem contribuir na criação de protocolos para indicação de histeroscopia diagnóstica em mulheres menopausadas.

\section{Referências}

ACOG (American College of Obstetricians and Gynecologists). (2009). ACOG Committee Opinion No. 440: The role of transvaginal ultrasonography in the evaluation of postmenopausal bleeding. Obstetrics \& Gynecology, 114, 409-411.

Accorsi Neto, A., Gonçalves, W., Mancini, S., Soares Junior, J., Haidar, M., Lima, G., et al. (2003). Comparação entre a histerossonografia, a histeroscopia e a histopatologia na avaliação da cavidade uterina de mulheres na pós-menopausa. Revista Brasileira de Ginecologia e Obstetrícia, $25,667-72$.

American Cancer Society (2017). Cancer Facts \& Figures. American Cancer Society, p. 29.

Baracat, E. C., Soares Júnior, J. M., Haidar, M. A., \& de Lima, G. R. (2003). Aspectos reprodutivos no climatério. In C .E. Fernandes (Eds.), Menopausa: diagnóstico e tratamento (1st ed., p.125-129). Segmento.

Berek, J. S. (2014). Tratado de Ginecologia (15th ed). Guanabara Koogan.

Borges P. (2019). Correlação ultrassonográfica e histeroscópica no diagnóstico de pólipos endometriais em mulheres na pós-menopausa [Tese, Faculdade de Medicina de Botucatu].

Branco, H. K. M. S. M. C., Depes, D. B., Baracat, F. F., Lippi, U. G., Takahashi, W. H., \& Lopes, R. G. C. (2008). Achados histeroscópicos em pacientes na pós-menopausa com espessamento endometrial à ultra-sonografia. Einstein, 6 (3), 287-292.

Breijer, M., Peeters, J., Opmeer, B., Clark, T., Verheijen, R., Mol, B., et al. (2012). Capacity of endometrial thickness measurement to diagnose endometrial carcinoma in asymptomatic postmenopausal women: a systematic review and meta-analysis. Ultrasound in Obstetrics \& Gynecology, 40 (6), 621-629.

Burbos, N., Musonda, P., Giarenis, I., Shiner, A., Giamougiannis, P., Morris, E., et al. (2010). Predicting the risk of endometrial cancer in postmenopausal women presenting with vaginal bleeding: the Norwich DEFAB risk assessment tool. British Journal of Cancer. 102 (8), $1201-1206$.

Campaner, A., Piato, S., Ribeiro, P., Aoki, T., Nadais, R., \& Prado, R. (2004). Achados histeroscópicos em mulheres na pós-menopausa com diagnóstico de espessamento endometrial por ultra-sonografia transvaginal. Revista Brasileira de Ginecologia e Obstetrícia, 26 (1), 53-58. 
Costa, H. L. F. F., \& Costa, L. O. B. F. (2008). Histeroscopia na menopausa: análise das técnicas e acurácia do método. Revista Brasileira de Ginecologia e Obstetrícia, 30 (10), 524-530.

Donadio, N., Albuquerque, Neto, L. (2001). Consenso brasileiro em videoendoscopia ginecológica. FEBRASGO.

Elfayomy, A., Habib, F., \& Alkabalawy, M. (2012). Role of hysteroscopy in the detection of endometrial pathologies in women presenting with postmenopausal bleeding and thickened endometrium. Archives of Gynecology and Obstetrics. 285 (3), $839-843$.

Estrela, C. (2018). Metodologia Científica: Ciência, Ensino, Pesquisa. Editora Artes Médicas.

Gambacciani, M., Monteleone, P., Ciaponi, M., Sacco, A., \& Genazzani, A. (2004). Clinical usefulness of endometrial screening by ultrasound in asymptomatic postmenopausal women. Maturitas, 48 (4), 421-424.

INCA (Instituto Nacional de Câncer José Alencar Gomes da Silva). (2015). Estimativa 2016: incidência de câncer no Brasil. INCA, 49-50.

Labastida, R. N. (1990). Tratato y atlas de histeroscopia. Salvat.

Lo, K., \& Yuen, P. (2000). The Role of Outpatient Diagnostic Hysteroscopy in Identifying Anatomic Pathology and Histopathology in the Endometrial Cavity. The Journal of the American Association of Gynecologic Laparoscopists, 7(3), 381-385.

Machado, M., Pina, H., \& Matos, E. (2003). Acurácia da histeroscopia na avaliação da cavidade uterina em pacientes com sangramento uterino pósmenopausa. Revista Brasileira de Ginecologia e Obstetrícia. 25 (4), 237-241.

Metello, J., Relva, A., Milheras, E., \& Colaço, J. (2008). Eficácia diagnóstica da histeroscopia nas metrorragias pós-menopausa. Acta Médica Portuguesa, 21 (5), 483-488.

Pop-Trajkovic-Dinic, S., Ljubic, A., Kopitovic, V., Antic, V., Stamenovic, S., \& Trninic-Pjevic, A. (2013). The role of hysteroscopy in diagnosis and treatment of postmenopausal bleeding. Vojnosanitetski pregled. 70 (8), 747-750.

Sarvi, F., Alleyassin, A., Aghahosseini, M., Ghasemi, M., \& Gity, S. (2016). Hysteroscopy: A necessary method for detecting uterine pathologies in postmenopausal women with abnormal uterine bleeding or increased endometrial thickness. Journal of Turkish Society of Obstetric and Gynecology, 13 (4), 183 188.

Silva D. (2015). Comparação entre os achados ecográficos, histeroscópicos e o anatomopatológico de pacientes pós menopausa encaminhadas para o ambulatório de histeroscopia [Tese, Universidade Federal de Ciências da Saúde de Porto Alegre].

Smith-Bindman, R., Weiss, E., \& Feldstein, V. (2004). How thick is too thick? When endometrial thickness should prompt biopsy in postmenopausal women without vaginal bleeding. Ultrasound in Obstetrics and Gynecology. 24(5),558-565.

Takacs, P., De Santis, T., Nicholas, M., Verma, U., Strassberg, R., \& Duthely, L. (2005). Echogenic Endometrial Fluid Collection in Postmenopausal Women Is a Significant Risk Factor for Disease. Journal of Ultrasound in Medicine, 24 (11), 1477-1481.

Tinelli, R., Tinelli, F. G., Cicinelli, E., Malvasi, A., \& Tinelli, A. (2008). The role of histeroscopy with eye-directed biopsy in postmenopausal women with uterine bleeding and endometrial atrophy. Menopause, 15 (4), 737-42.

Topçu, H., Taşdemir, Ü., İslimye, M., Bayramoğlu, H., \& Yılmaz N. (2015). The clinical significance of endometrial fluid collection in asymptomatic postmenopausal women. Climacteric. 18 (5), 733-736.

World Health Organization. (2017). World Health Organization Statistics Information System. WHO.

Worley, M., Dean, K., Lin, S., Caputo, T., \& Post, R. (2011). The significance of a thickened endometrial echo in asymptomatic postmenopausal patients. Maturitas, 68 (2), 179-181.

Yela, D. A., Ravacci, S. H., Monteiro, I. M. U., Pereira, K. C. H. M., \& Gabiatti, J. R. E. (2009). Comparação do ultrassom transvaginal e da histeroscopia ambulatorial no diagnóstico das doenças endometriais em mulheres menopausadas. Revista da Associação Médica Brasileira, 55 (5), $553-556$. 